\title{
Synthesis and Polycondensation of a Cyclic Oligo(phenylsilsesquioxane) as a Model Reaction for the Formation of Poly(silsesquioxane) Ladder Polymer
}

\author{
Eung-Chan LeE and Yoshiharu Kimura \\ Department of Polymer Science and Engineering, Kyoto Institute of Technology, \\ Matsugasaki, Sakyo-ku, Kyoto 606-8585, Japan
}

(Received March 9, 1998)

\begin{abstract}
A tetracyclo(phenylsilsesquioxane) (T4) was first synthesized by the oligomerization of the low molecular weight hydrolysate of trichlorophenylsilane (TCP) by catalysis of the weak base, $\mathrm{NaHCO}_{3}$, followed by $\mathrm{KOH}$-catalyzed polycondensation in toluene to form poly(phenylsilsesquioxane) (PPQS). The rate of polycondensation of T4 was much slower than that of direct polycondensation of the TCP hydrolysate under identical conditions. The molecular weight of the former product $\left(M_{n}=35800\right)$ was significantly lower than that of the latter product $\left(M_{n}=110000\right)$. X-Ray scatterings of the PPQS prepared by both processes were slightly different, probably due to the difference in structural regularity or branched structure. These data suggest that the polycondensations of T4 and the TCP hydrolysate should be driven through different reaction mechanisms, although the formation of the double strand ladder chain in the produced PPQS should be common to both KEY WORDS Trichlorophenylsilane / Hydrolytic Polycondensation / Tetra(phenylsilsesquioxane) / Silanol Capping / Poly(phenylsilsesquioxane) / Ladder Polymer /
\end{abstract}

Poly(phenylsilsesquioxane) (PPQS) with the empirical composition of $\left(\mathrm{C}_{6} \mathrm{H}_{5} \mathrm{SiO}_{1.5}\right)_{x}{ }^{1-7}$ has been prepared under various conditions to control its structure and properties which have not yet been well characterized. Recently, ${ }^{8}$ we re-investigated the hydrolysis of trichlorophenylsilane (TCP) in a water/toluene solvent system and found that low molecular weight TCP hydrolysates can be isolated from the aqueous layer and their base-catalyzed polycondensation affords a high molecular weight PPQS. PPQS thus obtained was found to be highly crystalline, having a small number of defects in the double-chain ladder structure. ${ }^{9}$ These data suggested that the ladder structure of PPQS can be well controlled as long as the molecular weight TCP hydrolysates are used in the base-catalyzed polycondensation, preferably the monomeric phenylsilanetriol (PST), although the formation mechanism is unknown.

In 1963, Brown reported that the base-catalyzed equilibration of a medium molecular weight PPQS in dilute solution produced the low molecular weight polycyclic species rather than a very high molecular weight soluble ladder polymer, ${ }^{10,11}$ usually obtained in solid system at high temperature. The polycyclic species were believed to be intermediates such as T8 (an octamer with cubic structure) and T16 (a hexadecamer with two cage-like components of T8) which are both composed of a cyclic tetramer T4 with eight-membered ring. Based on this finding it was thought that the polycondensation of the TCP hydrolysates should proceed via the formation of T4 to yield PPQS. However, no experimental evidence for this has been obtained. It has also been controversial whether the base-treatment of PPQS, having the stable double strand structure, undergoes depolymerization in solution by breaking the T4 unit. It is thus important to know whether T4 is formed as the intermediate during the polycondensation of the TCP hydrolysates.

In the present investigation an oligo(phenylsilsesquioxane) was synthesized by the oligocondensation of the low molecular weight TCP hydrolysate by the catalysis of the weak base, $\mathrm{NaHCO}_{3}$, instead of the strong base,
$\mathrm{KOH}$, generally used for polycondensation. It was then subjected to the $\mathrm{KOH}$-catalyzed polycondensation to form PPQS with medium molecular weight (Scheme 1). This reaction may give not only an insight into the formation mechanism of PPQS, but also structural information on the produced polymer.

\section{EXPERIMENTAL}

\section{Materials}

TCP and chlorotrimethylsilane (CTM) were supplied by Nacalai Tesque Co. (Kyoto) and purified by distillation. Toluene and cyclohexane were distilled over sodium metal before use. Other solvents and reagents were commercially supplied and used without purification.

\section{Measurements}

${ }^{29} \mathrm{Si}$ NMR spectra were measured at $99.3 \mathrm{MHz}$ on a Brucker ARX-500 spectrometer in chloroform- $d_{1}$ and toluene- $d_{8}$. A capillary glass tube containing pure tetramethylsilane (TMS) was put into an NMR sample tube made of glass $(5 \mathrm{~mm} \phi)$ as the in situ external standard together with sample solution. Measurement was done at $21^{\circ} \mathrm{C}$ by spinning the sample tube at 5000
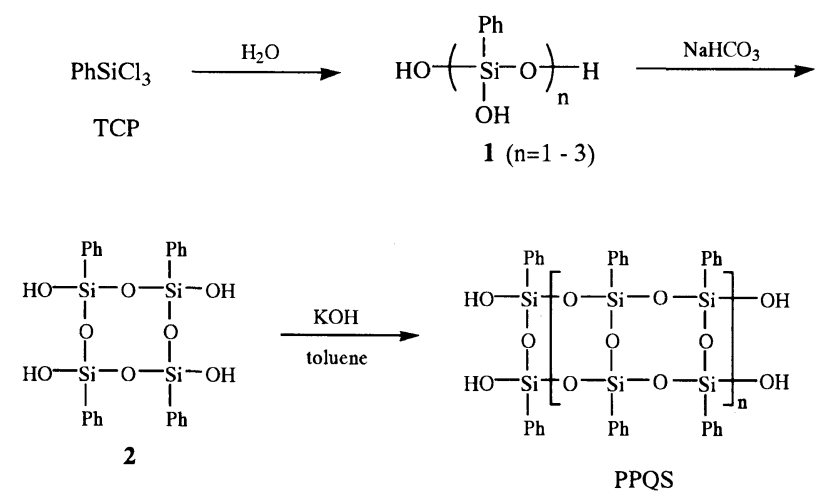

Scheme 1. 
$\min ^{-1} .{ }^{1} \mathrm{H}$ NMR spectra were measured at $200 \mathrm{MHz}$ on a Varian XL-200 spectrometer in $\mathrm{CDCl}_{3}$ with the solvent peak as the internal reference. The numberaverage molecular weight $\left(M_{n}\right)$ and molecular weight distribution $\left(M_{w} / M_{n}\right)$ were determined by gel permeation chromatography (GPC). The analyzer was composed of Shimadzu LC-10A pump, Shodex RI SE-31 RI detector, Shimadzu C-R7A Chromatopac data processor, and Shodex DEGAS KT-16 degassor. A combination of two polystyrene gel columns of Tosoh TSK gel G4000 $\mathrm{H}_{8}$ and $\mathrm{G} 2500 \mathrm{H}_{8}$ was used with chloroform as the eluent at $35^{\circ} \mathrm{C}$. The limited exclusion molecular weight was $7 \times 10^{5} \mathrm{Da}$ and molecular weight was calibrated according to polystyrene standards. FT-IR spectra were recorded on a JASCO FT/IR-5300 spectrophotometer by the $\mathrm{KBr}$ pellet method. Wide angle X-ray scattering (WAXS) was measured on a JEOL DX-GE-E model diffractometer with Ni-filtered $\mathrm{Cu}-K$ radiation with wave length of $1.542 \AA$ generated at $40 \mathrm{kV}$ and $20 \mathrm{~mA}$. The molecular weights of the oligocondensate and siloxyl-capping products were measured by cryoscopy. This measurement was done for a benzene solution of the sample at a concentration of $0.88-1.98 \mathrm{~g}$ per $100 \mathrm{~g}$ benzene.

\section{Preparation of TCP Hydrolysate}

The procedure was as reported previously. ${ }^{8}$ After a TCP solution in toluene had been added to excess water at $0^{\circ} \mathrm{C}$, the aqueous and toluene layers were separated. As soon as the $\mathrm{pH}$ of the aqueous layer was adjusted to 4 with $\mathrm{NaHCO}_{3}$, the hydrolysate with $M_{n}=170$ precipitated out as white crystals which were filtered and dried in vacuo. ${ }^{1} \mathrm{H}$ NMR $\left(\mathrm{CDCl}_{3}\right): \delta 7.11-7.8(\mathrm{~m}$, $\left.\mathrm{C}_{6} \mathrm{H}_{5}-\right), 2.2-2.8$ (s, Si-OH) ppm; IR (KBr): $3600-3200$ $(\mathrm{Si}-\mathrm{Ph}), 3080-2940\left(v_{\mathrm{C}-\mathrm{H}}\right), 1435(\mathrm{Si}-\mathrm{Ph}), 1050(\mathrm{O}-\mathrm{Si}$ $\mathrm{Ph}), 750$, and $700(\mathrm{Ph}) \mathrm{cm}^{-1} ;{ }^{29} \mathrm{Si} \mathrm{NMR}\left(\mathrm{CDCl}_{3}\right): \delta$ $-64.1 \mathrm{ppm}$. When the filtrate was kept at $0^{\circ} \mathrm{C}$ overnight, the precipitates with $M_{n}=300$ deposited out as precipitates which were isolated by filtration and dried in vacuo.

\section{Preparation of Oligocondensate}

$25 \mathrm{~g}$ of TCP hydrolysates with $M_{n}=170$ were mixed with $1.25 \mathrm{~g}(5 \mathrm{wt} \%)$ of $\mathrm{NaHCO}_{3}$ in $1600 \mathrm{ml}$ of toluene. The mixture was stirred at $3^{\circ} \mathrm{C}$ for $48 \mathrm{~h}$. The obtained mixture was filtered and condensed to $1 / 4$ in volume by evaporating the solvent in vacuum to yield crystalline precipitates. The precipitates were collected by filtration and dried (yield: $13 \mathrm{~g}(57 \%)$ ). Some of these crystalline products was then subjected to recrystallization from chloroform. After 3 months, white shiny platelet-like crystals were obtained. ${ }^{1} \mathrm{H}$ NMR $\left(\mathrm{CDCl}_{3}\right): \delta 7.11-7.8$ $\left(\mathrm{m}, \mathrm{C}_{6} \mathrm{H}_{5}-\right), 2.2-2.8$ (s, Si-OH) ppm; IR (KBr): $3600-3200(\mathrm{Si}-\mathrm{Ph}), 3080-2940\left(v_{\mathrm{C}-\mathrm{H}}\right), 1435(\mathrm{Si}-\mathrm{Ph})$, $1050(\mathrm{O}-\mathrm{Si}-\mathrm{Ph}), 1150(\mathrm{O}-\mathrm{Si}), 750$, and $700(\mathrm{Ph}) \mathrm{cm}^{-1}$; ${ }^{29} \mathrm{Si} \mathrm{NMR}\left(\mathrm{CDCl}_{3}\right): \delta-64.1 \mathrm{ppm}$.

\section{Polycondensation}

$5.0 \mathrm{~g}$ crystalline oligocondensate obtained above and $5.0 \mathrm{mg} \mathrm{KOH}(0.1 \mathrm{wt} \%$ relative to the hydrolysate) were mixed with $38 \mathrm{ml}$ toluene. The mixture was heated at the refluxing temperature of toluene for $16 \mathrm{~h}$ with removing water from the azeotropically distilled tolu- ene/water mixture in a Dean-Stark tube. The viscous solution finally obtained was poured into large excess of methanol to precipitate the condensation product. The product was collected by filtration and dried in vacuo at $150^{\circ} \mathrm{C}$ for $10 \mathrm{~h} .{ }^{1} \mathrm{H}$ NMR $\left(\mathrm{CDCl}_{3}\right): \delta 7.11-7.8(\mathrm{~m}$, $\left.\mathrm{C}_{6} \mathrm{H}_{5}-\right)$ ppm; IR (KBr): $3600-3200(\mathrm{Si}-\mathrm{Ph}), 3080-2940$ $\left(v_{\mathrm{C}-\mathrm{H}}\right), 1435(\mathrm{Si}-\mathrm{Ph}), 1050(\mathrm{O}-\mathrm{Si}-\mathrm{Ph}), 1150(\mathrm{O}-\mathrm{Si}), 750$, and $700(\mathrm{Ph}) \mathrm{cm}^{-1} ;{ }^{29} \mathrm{Si} \mathrm{NMR}\left(\mathrm{CDCl}_{3}\right): \delta-83.7 \mathrm{ppm}$.

\section{Siloxyl-Capping Reaction}

$0.33 \mathrm{~g}$ crystalline oligocondensate and $0.17 \mathrm{~g}$ triethylamine were dissolved in $10 \mathrm{ml}$ cyclohexane. With this solution stirred, a solution of $0.13 \mathrm{~g}$ CTM in $10 \mathrm{ml}$ cyclohexane was added dropwise, and the mixture was stirred for another $90 \mathrm{~min}$. Care was taken to prevent the reaction temperature from rising above $20^{\circ} \mathrm{C}$. The yellow sticky precipitates formed in the mixture were separated by decantation and the solution was evaporated to dryness to obtain a solid product (yield: $0.1 \mathrm{~g}$ $(30 \%))$ which was recrystallized from chloroform to obtain large crystals. ${ }^{1} \mathrm{H}$ NMR $\left(\mathrm{CDCl}_{3}\right): \delta 7.11-7.8$ $\left(\mathrm{m}, \mathrm{C}_{6} \mathrm{H}_{5}-\right.$ ) ppm; IR (KBr): $3080-2940\left(v_{\mathrm{C}-\mathrm{H}}\right), 1435$ $(\mathrm{Si}-\mathrm{Ph}), 1620,1260,830(\mathrm{Si}-\mathrm{Me}), 1050(\mathrm{O}-\mathrm{Si}-\mathrm{Ph}), 1150$ $(\mathrm{O}-\mathrm{Si}), 750$, and $700(\mathrm{Ph}) \mathrm{cm}^{-1} ;{ }^{29} \mathrm{Si} \mathrm{NMR}\left(\mathrm{CDCl}_{3}\right): \delta$ $-78(\mathrm{O}-\mathrm{Si}-\mathrm{Ph}),-43.0\left(\mathrm{O}-\mathrm{Si}-\mathrm{Me}_{3}\right) \mathrm{ppm} ;{ }^{13} \mathrm{C} \mathrm{NMR}$ $\left(\mathrm{CDCl}_{3}\right): \delta 138.6$ (ipso carbon of $\mathrm{C}_{6} \mathrm{H}_{5}$ ), 136.9 (ortho carbons of $\mathrm{C}_{6} \mathrm{H}_{5}$ ), 132.7 (meta carbons of $\mathrm{C}_{6} \mathrm{H}_{5}$ ), 128.7 (para carbon of $\left.\mathrm{C}_{6} \mathrm{H}_{5}\right), 0.42\left(\mathrm{Si}-\mathrm{Me}_{3}\right) \mathrm{ppm}$.

\section{RESULTS AND DISCUSSION}

\section{Structure of the Crystalline Oligocondensate}

The TCP hydrolysate 1 with $M_{n}=170$ (consisting mainly of PST: $1(n=1))$ was subjected to oligocondensation by catalysis of the weak base, $\mathrm{NaHCO}_{3}$, in toluene at low temperature. The condensation product was obtained as crystals and purified by recrystallization from chloroform although crystal forms of the products appeared polymorphic. Figure 1 shows the ${ }^{1} \mathrm{H}$ NMR spectrum of the recrystallized oligocondensate compared with that of the starting TCP hydrolysate. In the former, two simple signals due to the phenyl group are observed around $\delta 7.2$ and $7.8 \mathrm{ppm}$, and the siloxyl signal, around $\delta 2.5 \mathrm{ppm}$. The integral ratio of the phenyl $/ \mathrm{siloxyl}$ signals was almost $5 / 1$ for the oligocondensate instead of $5 / 3$ for the TCP hydrolysate (1). ${ }^{9}$ Figure 2 shows the ${ }^{29} \mathrm{Si} \mathrm{NMR}$ spectra of the oiligocondensate and the starting hydrolysate. The oligocondensate shows a single signal at $\delta-66.4 \mathrm{ppm}$ while the TCP hydrolysate one signal at slightly lower magnetic field $(\delta-64.1 \mathrm{ppm}){ }^{9}$ Observation of the sole signal at relatively higher magnetic field for the oligocondensate suggested that this product should have a very simple structure composed preferentially of $\mathrm{T}_{2}$ units. In an open chain structure, the signals due to the $T_{1}$ and $T_{2}$ units should appear as in the case of low molecular weight oligomers. ${ }^{8,9}$ (Here, $T_{i}$ $(i=0-3)$ denotes the specimen that has $i$ siloxane bonds and $(3-i)$ siloxyl groups with one phenyl side group.) The IR spectrum of the oligocondensate supported the presence of siloxyl and siloxane groups (see EXPERIMENTAL). The molecular weight of the oligocondensate was $553 \mathrm{Da}$ as measured by cryoscopy, this being consistent with the theoretical molecular weight of a 
a)

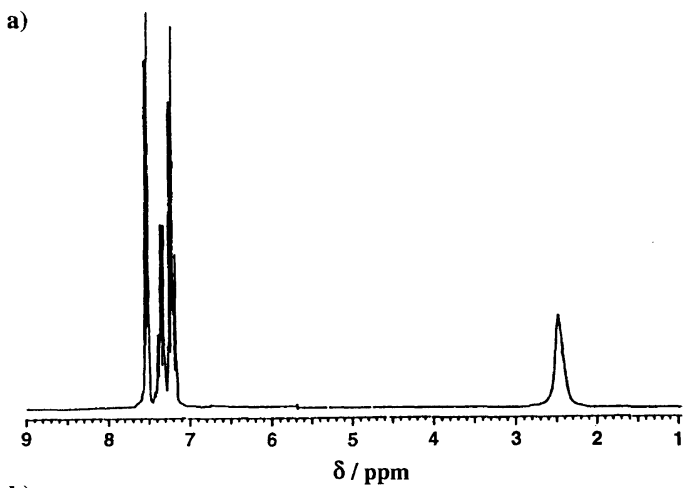

b)

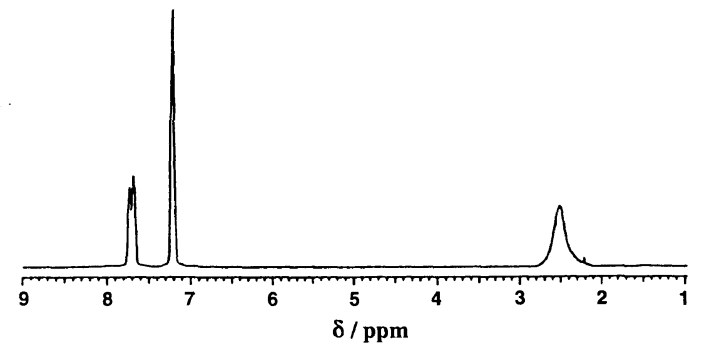

Figure 1. ${ }^{1} \mathrm{H}$ NMR spectra of a) the TCP hydrolysate and b) the oligocondensate (in $\mathrm{CDCl}_{3}$ ).
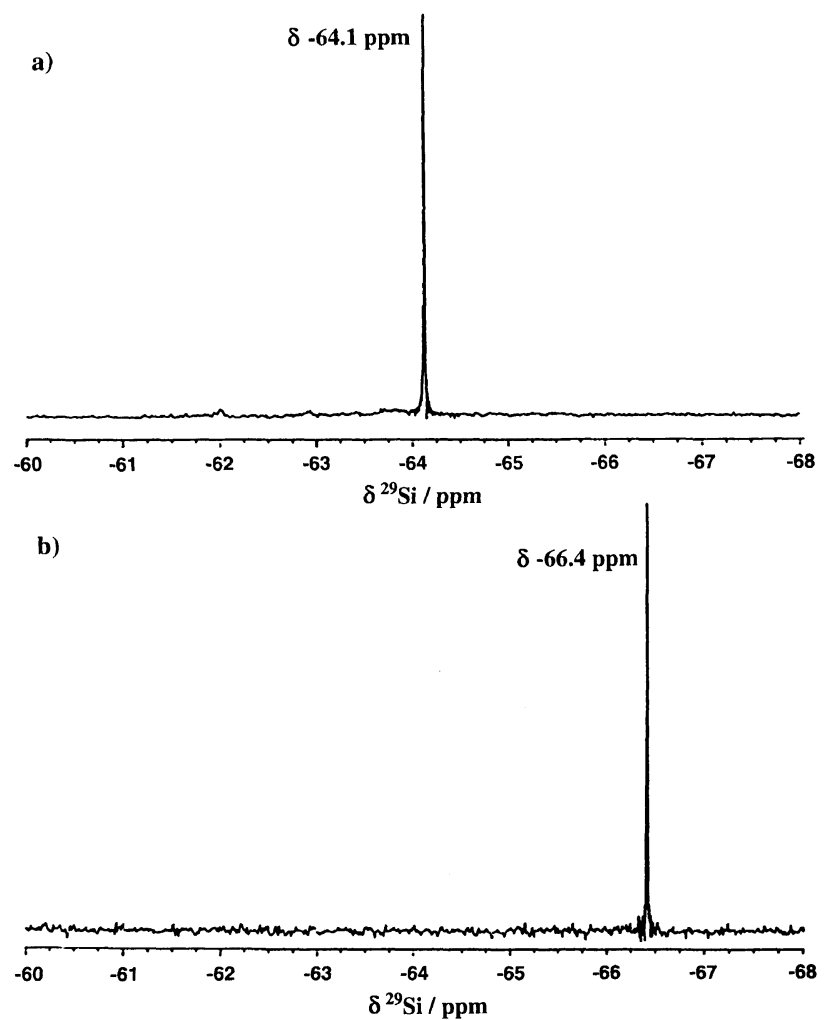

Figure 2. ${ }^{29} \mathrm{Si}$ NMR spectra of a) the TCP hydrolysate and b) the oligocondensate (in $\mathrm{CDCl}_{3}$ ).

cyclic tetramer consisting of $[\mathrm{PhSi}(\mathrm{O}) \mathrm{OH}]_{4}$ (Table I) consisting only of $\mathrm{T}_{2}$ unit. This value was comparable to the average molecular weight determined by GPC. Based on these data the oligocondensate was identified as the cyclic oligomer of T4 (2).

To support the structure of $\mathrm{T} 4$, the oligocondensate was subjected to siloxyl-capping reaction with CTM (Scheme 2). ${ }^{8}$ The capping product could be recrystallized into larger crystals for which a typical Bragg pattern
Table I. Molecular weights of compounds

\begin{tabular}{lcccc}
\hline \multicolumn{1}{c}{ Sample } & Theoretical Cryoscopy & \multicolumn{2}{c}{ GPC } \\
& & & $M_{n} / \mathrm{Da}$ & $M_{w} / M_{n}$ \\
\hline TCP hydrolysate & 156.62 & - & 170 & 1.1 \\
Oligocondensate & 552.74 & 553 & 640 & 1.0 \\
Silanol-capping compound & 841.45 & 841 & 870 & 1.0 \\
\hline
\end{tabular}

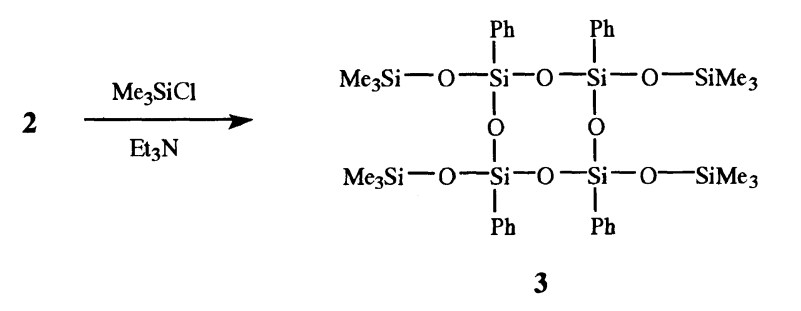

Scheme 2.

a)
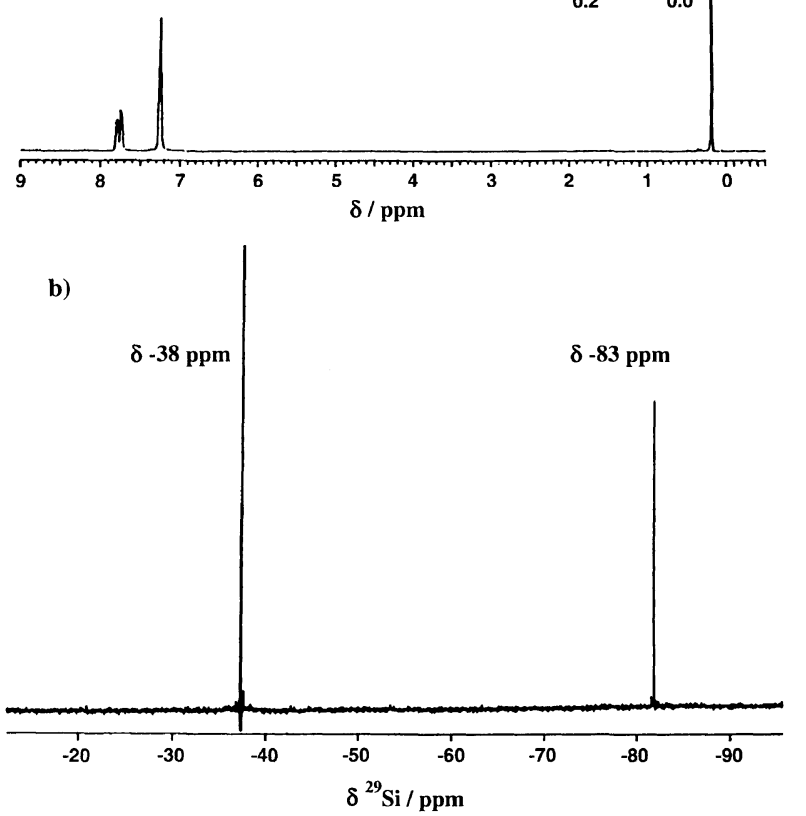

Figure 3. a) ${ }^{1} \mathrm{H}$ and b) ${ }^{29} \mathrm{Si} \mathrm{NMR}$ spectra of the siloxyl-capping product of the oligocondensate (in $\mathrm{CDCl}_{3}$ ). ${ }^{1} \mathrm{H}$ signal at $\delta 0.17 \mathrm{ppm}$ is widened in a).

could be observed by X-ray diffraction. The ${ }^{1} \mathrm{H}$ and ${ }^{29} \mathrm{Si}$ NMR spectra are shown in Figure 3. The phenyl proton signals to those of the original oligocondensate (Figure 1) and the siloxyl signal are replaced by the $\mathrm{CH}_{3}-\mathrm{Si}$ signal detected as a singlet at $\delta 0.2 \mathrm{ppm}$. The integral ratio of both signals was just $5 / 9$, indicating $1 / 1$ ratio for $\mathrm{C}_{6} \mathrm{H}_{5} /\left(\mathrm{CH}_{3}\right)_{3} \mathrm{Si}$. The silicon signal due to the $\left(\mathrm{CH}_{3}\right)_{3}-\mathrm{Si}$ is shown at $\delta-43.0 \mathrm{ppm}$, while that of the backbone units, at $-78.0 \mathrm{ppm}$. The high-field shift of the latter 

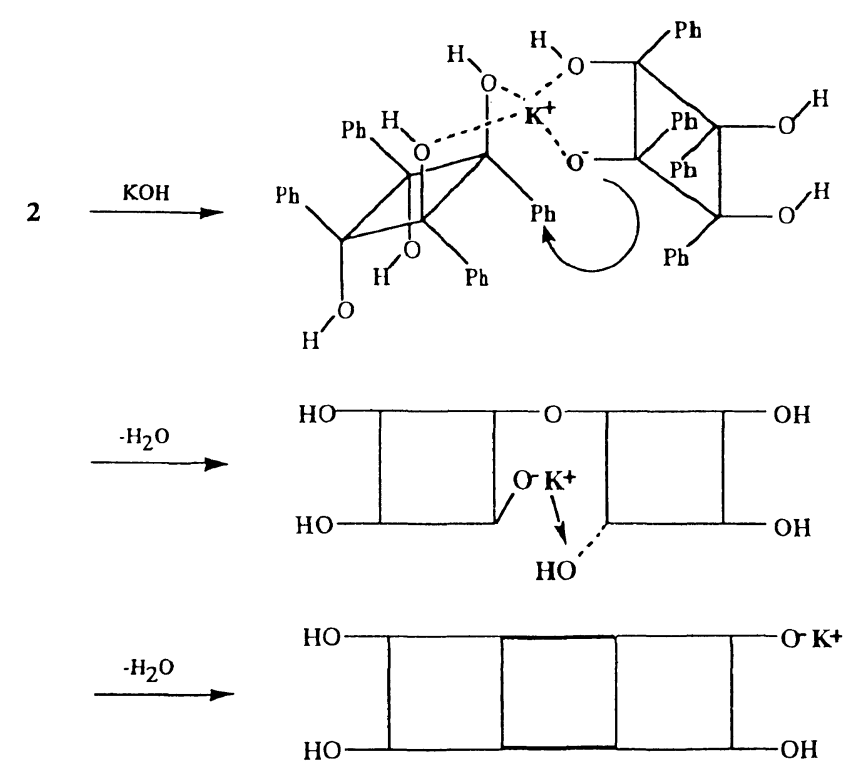

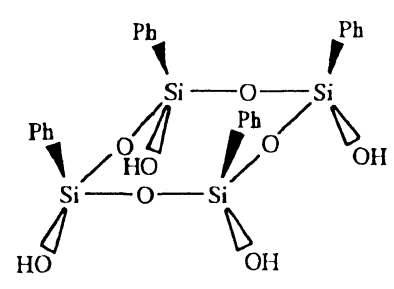

$2 \mathbf{a}$

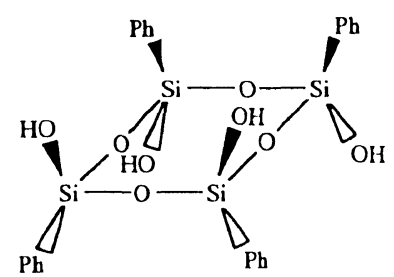

$2 c$
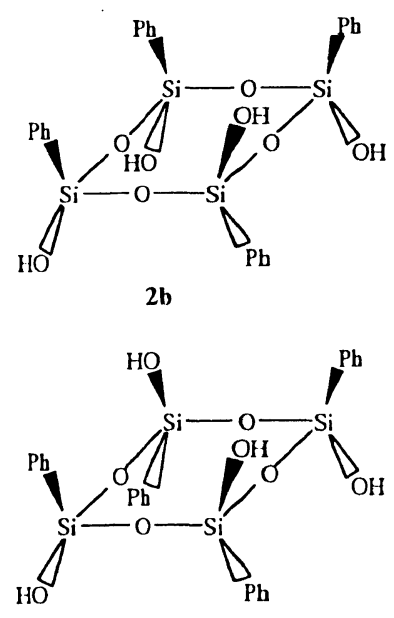

2d
Scheme 3.

signal from $-66.4 \mathrm{ppm}$ supported the quantitative transformation of $\mathrm{T}_{2}$ units to $\mathrm{T}_{3}$ units after the siloxyl capping reaction. IR data also supported the transformation of the siloxyl into the trimethylsilyloxyl group. The cryoscopic molecular weight of the capping product was 840 (Table I), which is consistent with the theoretical value of the trimethylsilylated T4 (3). These data well support the structures of $\mathbf{2}$ and $\mathbf{3}$ for the oligocondensate and its capping product, respectively.

The crystallinity of the T4 oligocondensate thus obtained was not very high, probably because it should have four configurational isomers $\mathbf{2 a - d}$ (Scheme 3) depending on the arrangement of the phenyl substituents on the ring. ${ }^{12}$ In the present experiment, recrystallization of the capping product $\mathbf{3}$ gave needle-like crystals in a relatively good yield. This compound is thought to comprise only one of the above four isomeric entities. The singlet signal observed for the $\left(\mathrm{CH}_{3}\right)_{3}-\mathrm{Si}$ protons suggests that the isomer has a symmetric configuration with environmentally equivalent siloxyl groups with respect to the ring plane. Therefore, the structure $\mathbf{2 b}$ is eliminated, and either all-cis (2a), cis-trans (2c), or all trans $\mathbf{( 2 d )}$ configuration is possible for the microstructure of 2 for which further identification was difficult. At present, we think that the original oligocondensate may contain the four isomers at a ratio depending on their formation probability and that only the major component (derived from either $\mathbf{2 a}, \mathbf{2 c}$, or $\mathbf{2 d}$ ) can be crystallized out, particularly so after the siloxyl capping reaction.

\section{Polycondensation of the Oligocondensate}

The oligocondensate, identified as T4, was subjected to polycondensation by the catalysis of $\mathrm{KOH}$ in refluxing toluene (Scheme 1). Figure 4 shows the time-dependent changes in the GPC curve of the resulting polycondensate under different polycondensation conditions. At a catalyst/oligocondensate ratio of 0.1 in wt\% (Figure 4a), the peaks centered at $M_{n}=830$, which corresponds to the oligocondensate, were replaced by the peak at $M_{n}=47300$ after $24 \mathrm{~h}$. The peak shifted to higher molecular weight region with reaction time. After $48 \mathrm{~h}$, the average molecular weight reached $53800 \mathrm{Da}$. Since in direct polycondensation of the TCP hydrolysate (Figure 4c) the average molecular weight reached $110000 \mathrm{Da}$ after much shorter reaction time of $16 \mathrm{~h}$ at the same catalyst ratio, ${ }^{9}$ the reaction rate is much slower in the polycondensation of the oligocondensate. When the oligocondensate was reacted by the catalysis of $0.01 \mathrm{wt} \%$ $\mathrm{KOH}$ (Figure $4 \mathrm{~b}$ ), the original peak at $M_{n}=830$ was replaced by the peak centered at $M_{n}=1790$ whose molecular weight was twice that of the original peak value. The latter was then replaced gradually by the peaks with higher $M_{n}$ with the reaction time passing, although the consumption of the oligocondensate was much slower than that at a catalyst ratio of $0.1 \mathrm{wt} \%$. This behavior supports a typical step-growth polymerization mechanism for this polycondensation. Since the time-dependent shift of the polycondensate peak was faster than the decrease of the oligocondensate peak, the rate of condensation should be faster for the polycondensates with higher molecular weight. In direct polycondensation of the TCP hydrolysate (Figure 4c), the oligocondensate (sharp peak at $M_{n}=830$ ) and polycondensate (broad peak) were formed in the initial stage, and the content of the oligocondensate increased up to $8 \mathrm{~h}$ and decreased thereafter, resulting in sharp increase in the molecular weight of the polycondensate. This suggests that the direct polycondensation of the TCP hydrolysate involves a reaction mechanism different from that for the oligocondensate.

\section{Difference in Structure Regularity of PPQS}

The ${ }^{29} \mathrm{Si}$ NMR spectrum of the polycondensates (PPQS) prepared from the oligocondensate showed a sharp signal at $\delta-83.7 \mathrm{ppm}$ as with the one prepared from the TCP hydrolysate, which should be attributed to the $\mathrm{T}_{3}$ units exclusively formed in the polymers. The signal width at half height was much narrower in the former $(50 \mathrm{~Hz})$. Therefore, PPQS prepared from the oligocondensate should have a structure with higher regularity, at least in terms of the environmental homogeneity of each repeating unit.

Table II summarizes WAXS data (in $d$-spacing of the Debye-Scherrer rings) of the PPQS $\left(M_{n}=53800\right)$ prepared from the oligocondensate and the PPQS $\left(M_{n}=\right.$ 
a)

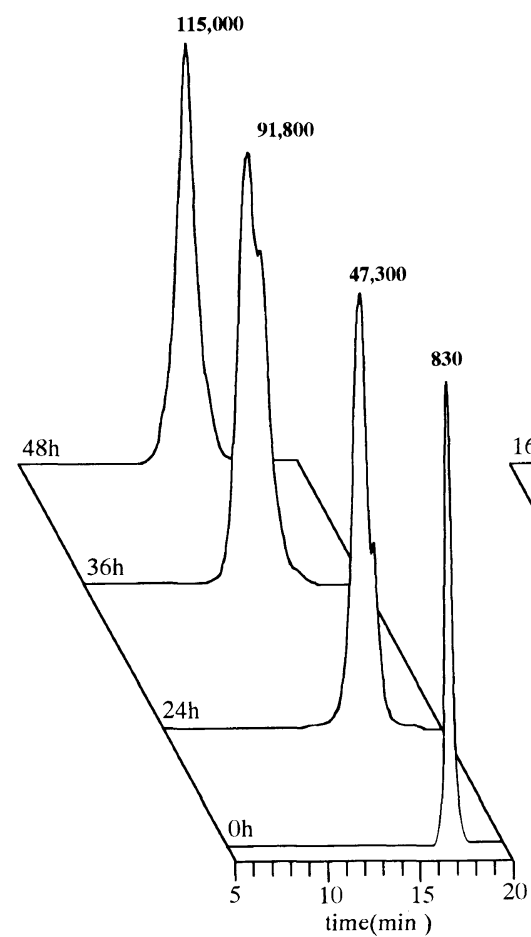

b)

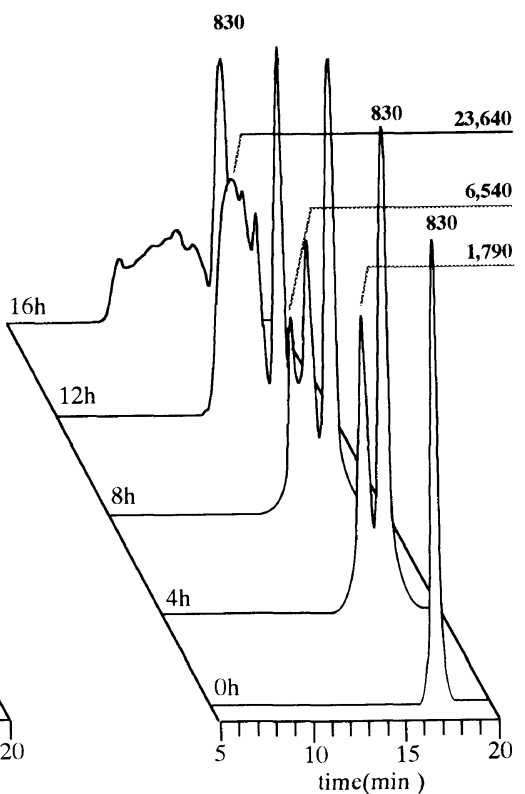

c)

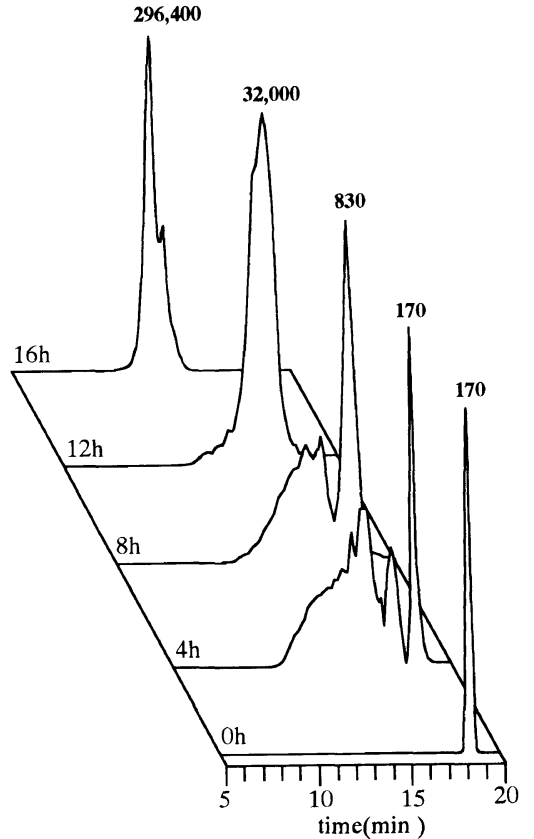

Figure 4. Changes in GPC of the condensation product with reaction time in the polycondensations of the oligocondensate in the presence of a) $0.1 \mathrm{wt} \% \mathrm{KOH}$, b) $0.01 \mathrm{wt} \% \mathrm{KOH}$, and c) the TCP hydrolysate in the presence of $0.1 \mathrm{wt} \% \mathrm{KOH}$. Numerical values above the peaks indicate number-average molecular weights of peak tops.

Table II. WAXS data $(d$-spacing $/ \AA)$ of PPQS and oligocondensate

\begin{tabular}{ccc}
\hline PPQS $^{\mathrm{a}}$ & PPQS $^{\mathrm{b}}$ & Oligocondensate \\
\hline $12.01(\mathrm{~s})^{\mathrm{c}}$ & $11.39(\mathrm{vs})$ & $16.31(\mathrm{vs})$ \\
$8.17(\mathrm{~m})$ & $9.52(\mathrm{~m})$ & $11.11(\mathrm{vs})$ \\
& & $6.31(\mathrm{w})$ \\
$4.90(\mathrm{~m})$ & $4.90(\mathrm{~m})$ & \\
$4.61(\mathrm{~s})$ & $4.66(\mathrm{~m})$ & $4.65(\mathrm{vs})$ \\
& $4.25(\mathrm{~m})$ & $4.25(\mathrm{w})$ \\
$3.58(\mathrm{w})$ & $3.58(\mathrm{~m})$ & $3.92(\mathrm{~s})$ \\
& & $3.58(\mathrm{w})$ \\
\hline
\end{tabular}

${ }^{a}$ Prepared from the oligocondensate (as-prepared). ${ }^{b}$ Prepared from the hydrolysate and heat-treated at $350^{\circ} \mathrm{C}$. ${ }^{\mathrm{c}} \mathrm{vs}$, very strong; s, strong; m, medium; w, weak.

110000) prepared from the TCP hydrolysate, which are compared with the powder pattern of the oligocondensate itself. Each PPQS showed a slightly different pattern, probably because of differences in microstructure and packing of the polymer chains. With regard to diffractions having short $d$-spacings, the asprepared PPQS prepared from the oligocondensate showed one strong diffraction at $d$-spacing of $4.61 \AA$ while the heat-treated PPQS prepared from the TCP hydrolysate showed two medium-strength diffractions at $d$-spacings of $4.66 \AA$ and $4.25 \AA$. Diffraction of the former appeared between the two diffractions of the latter. There is similarity between the diffraction patterns of the PPQS and original oligocondensate, although the oligocondensate does not show diffraction at $d$-spacing of $4.90 \AA$ and each diffraction is somewhat different in $d$-spacing. This suggests that cyclic units of $\mathrm{T} 4$, should be common to the two PPQS and oligocondensate in spite of differences in packing state. Since diffraction with medium $d$-spacings around $11-12 \AA$, considered to correspond to the side lengths $\left(e . g ., H_{\mathrm{Ph}}-H_{\mathrm{Ph}}\right)$ of the $\mathrm{T} 4$ ring, ${ }^{13}$ is significantly different, the phenyl arrangement should be varied in each compound. The similar diffraction intensity between the two PPQS suggests that the crystallinity should be almost identical. However, PPQS as-prepared from the hydrolysate (before the heat-treatment) showed a very weak diffraction at $d$ spacing of $4.6 \AA$. Therefore, we consider that the PPQS prepared directly from the oligocondensaste has a slightly more regular structure than the PPQS prepared from the hydrolysate. At present, the real microstructure of PPQS has not been determined spectrometrically.

\section{Mechanism of Polycondensation}

The above data show that oligocondensate T4 is formed through oligomerization of the low molecular weight hydrolysate (1) followed by cyclization. The preferential formation of the tetramers may be ascribed to the use of weak base which promotes dehydration of the siloxyl groups on the $T_{0}$ and $T_{1}$ units only to give the $T_{2}$ units by cyclization.

In the polycondensation of $\mathrm{T} 4$, dehydration among the $T_{2}$ siloxyl groups occurs stepwise to form dimers, trimers, tetramers, and so on. The rate of this polycondensation is much slower than the polycondensation of the original TCP hydrolysate, because the more reactive $T_{0}$ and $T_{1}$ siloxyl groups are absent in the units, since the starting $\mathrm{T} 4$ involves stereoisomers, and ring closure on the edges of the two rings may not easily occur. A possible condensation of the two cis-trans isomers (2c) is shown in Scheme 3. When the siloxylate anion formed on one of the siloxyl groups of the right ring attacks a siloxyl group of the left ring by the aid of 
$\mathrm{KOH}$ to form siloxane bonds, each adjacent siloxyl group on the two rings has an arrangement in which formation of another siloxane linkage is allowed to give a new ring. If similar reaction proceeds between the different isomers, such arrangement should not be allowed, retarding the cyclization. A chain rearrangement or isomerization is needed for suitable arrangement of the two siloxyl groups. ${ }^{11,14}$ This steric requirement may be the main reason for the slow rate of polycondensation of $\mathrm{T} 4$. The chain configuration of the produced PPQS should be random in terms of stereoregularity. However, its sterically crowded chain structure limits not only the substituent arrangement, but also the direction of chain extension, and the chain propagation should be allowed in such a way as to form a certain chain configuration by forcing the $T_{2}$ units to isomerize. Consequently, the sterically less hindered trans-syndiotactic configuration is formed predominantly over the cis-isotactic configuration to yield a crystalline PPQS, as reported previously. ${ }^{14}$

In the polycondensation of TCP hydrolysates, the reaction mechanism differs from the above. Since the polycondensation of the $T_{0}$ and $T_{1}$ siloxyls are promoted by the catalysis of strong base, the polymer tails may involve $T_{1}$ siloxyls, and the rate of polycondensation is much faster than that of condensation of T4. During the polycondensation of the hydrolysate $\mathrm{T} 4$ is formed and reacts with the polymer tails of the $T_{1}$ structure with a relatively faster rate. In this case the chain configuration is controlled by the steric requirements to form trans-syndiotactic sequence. ${ }^{9}$ Therefore, an intrinsically same polymer structure is realized in the polycondensations of T4 and the primary condensates of PST, although the sequence regularity is higher in the polycondensate of T4. Formation of such structure is prohibited if an oligomer having an irregular structure is used as a starting material usually formed by the acid-catalyzed condensation of the primary hydrolysate of TCP in aqueous solution. In the present experiment, no medium-sized polycyclic oligomer was formed at all during the polycondensation, probably because the reaction was conducted at a relatively higher concentration of $\mathrm{T} 4$. The polymer/polycyclics equilibrium proposed by Spurung and Brown et al. may not be considered in our system. The conversion of T4 to T8 is really a chain extension of the ladder, not the formation of a cuban-like polycyclic compound.

\section{CONCLUSIONS}

An oligocondensate was synthesized by dehydration of the low molecular weight TCP hydrolysate by the catalysis of a weak base, $\mathrm{NaHCO}_{3}$. The structure was identified as tetracyclo(phenylsilsesquioxane) (T4) . By $\mathrm{KOH}$-catalyzed polycondensation of this compound, crystalline PPQS with improved structure regularity was prepared. The rate of this polycondensation was much slower than that of the direct polycondensation of the TCP hydrolysate, and the molecular weight of the PPQS was significantly lower. These data suggest that the mechanism of polycondensation of the TCP hydrolysate differs from the coupling of the pre-formed T4, although the chain structure of both produced PPQS is similar to each other. A steric requirement of the bulky repeating units is to control the direction of the chain growth and even the chain configuration of PPQS to form transsyndiotactic sequence.

\section{REFERENCES}

1. H. Adachi, E. Adachi, S. Yamamoto, and H. Kanegae, Mat. Res. Soc. Symp. Proc., 227, 95 (1991).

2. F. Matsui, Kobunshi Kako, 39, 299 (1990).

3. A. Oikawa, S. Fukuyama, Y. Toneda, H. Harada, and T. Takada, J. Electrochem. Soc., 137, 3223 (1990).

4. H. Adachi, H. Kanegae, and Y. Tsuchiya, Denshi Zairyou, 135 (1990).

5. B. G. Bagley, W. E. Quinn, and C. R. Kurkjian, Polym. Eng. Sci., 29, 1197 (1989).

6. M. Ishiharaga, H. Kaneda, T. Chikaraishi, S. Tomita, I. Tanuma, and K. Naito, Sen'i Gakkai Symp. Prepr., B-11 (1992).

7. F. Survivet, T. M. Lan, J. P. Pascault, and Q. T. Pham, Macromolecules, 25, 4309 (1992).

8. E. C. Lee and Y. Kimura, Polym. J., 30, 234 (1998).

9. E. C. Lee and Y. Kimura, Polym. J., 29, 678 (1997).

10. K. Larsson, Arkiv Kemi, 16, 209 (1961).

11. M. M. Spurung and F. O. Guenther, J. Polym. Sci., 28, 17 (1958).

12. J. F. Brown, H. V. Lester, and P. I. Prescott, J. Am. Chem. Sci., 86, 1120 (1963)

13. K. A. Andrianov, G. L. Slonimsky, A. A. Zhdanov, D. Ya. Tsvankin, V. Yu. Levin, V. S. Papkov, Yu. P. Kvachev, and E. M. Belavtseva, J. Polym. Sci., 14, 1205 (1976).

14. J. F. Brown and L. H. Vogt, J. Am. Chem. Soc., 87, 4313 (1965). 